\title{
Intraparenchymal central nervous system Ewing's sarcoma. A case treated according to the modified Euro- EWING protocol
}

\author{
Marie-Amelyne Le Rouzic ${ }^{1}$, Julie Valduga ${ }^{1}$, Ludovic Mansuy ${ }^{1}$, Emmanuelle Schmitt ${ }^{2}$, \\ Olivier Klein ${ }^{3}$, Valérie Bernier ${ }^{4}$, Pascal Chastagner ${ }^{*}, 1$ \\ ${ }^{1}$ Department of Paediatric Hematology and Oncology, Children's University Hospital, Nancy, France; \\ ${ }^{2}$ Department of Paediatric Neuroradiology, University Hospital, Nancy, France; ${ }^{3}$ Department of \\ Paediatric Neurosurgery, Children's University Hospital, Nancy, France; ${ }^{4}$ Department of Radiotherapy, \\ Institut de Cancérologie en Lorraine, Nancy, France
}

\begin{abstract}
Ewing's sarcoma (ES) is the second most common malignant bone tumor in children and young adults. ES may also occur as a primary soft tissue neoplasm without involvement of the bone and is then referred to as extraosseous ES (EES). The paediatric central nervous system extraosseous Ewing's sarcoma (CNS-EES) is an extremely rare entity since less than 20 cases have been reported in literature. Moreover, only less than 10 cases correspond to brain parenchyma primary tumors. These cases have been often misdiagnosed with other varieties of primary brain tumors, particularly those which were called PNET (Primitive Neuro-Ectodermal Tumor). We present a case of true intraparenchymal CNS-EES and a comparison of epidemiology, behaviour, treatment, and prognosis of CNS-EES and intracranial PNET cases.
\end{abstract}

Keywords: extraosseous Ewing's sarcoma, central nervous system, surgery, chemotherapy, radiotherapy, child

\section{Introduction}

Ewing's sarcomas (ES) are small roundcell neoplasms, presumed to be neuroectodermal in origin, which occur most often in the bones in adolescents and young adults. While Ewing's sarcoma arising from the skull is rare, the intracranial parenchymal location and manifestation of the disease is extremely rare, and when present, this is often misdiagnosed with other varieties of primary brain tumors. We report a case of intracranial Ewing's sarcoma, which was initially suspected to be a central nervous system PNET (c-PNET) in an 8-year-old girl.

Received: October 2017; Accepted after review: November 2017; Published: December 2017.

${ }^{*}$ Corresponding author: Prof. Pascal Chastagner, Department of Pediatric Hematology and Oncology, Children's University Hospital, 11 Allée du Morvan, 54511 Vandoeuvre, France.

Email: p.chastagner@chru-nancy.fr

\section{Case Report}

An 8-year-old female presented to her physician in February 2013 with a 6-month history of headache, nausea, and vomiting, associated to behaviour disorders which had increased in intensity over the last two months. Her general and neurological status was normal. An MRI scan of the brain revealed a $\mathrm{T}-1$ hyperintense bilateral frontal mass $(85 \mathrm{x}$ $62 \times 66 \mathrm{~mm}$ ) with solid and cystic components and heterogeneous enhancement (Figure 1). A significant mass effect was detected. No bone extension was observed. No evidence of calcification was noted within the lesion on CT scan. She underwent left temporo-parietal craniotomy and partial resection of a very vascular tumor. Post-operative MRI scan revealed gross residual disease. Pathology revealed a small round blue cell tumor positive for CD99/MIC-2 antigen suggestive of Ewing's sarcoma. The diagnosis was confirmed by FISH analysis based on the presence of 
EWS/FLI1 fusion transcript and rearrangements of the EWS and FLI1 gene loci. Metastatic work-up including MRI of the spine, PET scan, CT scan of chest and abdomen, cerebrospinal fluid cytology, bone scan, bone marrow aspirate and biopsy, was negative. She then received 6 cycles of chemotherapy with vincristine, cyclophosphamide, and doxorubicin alternating with ifosfamide and etoposide
(EURO EWING sarcoma 2012). She had an almost complete resolution of tumor following these cycles of chemotherapy. She then underwent focal radiotherapy of 54 Gy (120 Gy b.i.d daily for 6 weeks) to the primary site and completed treatment with six more cycles of the TEMIRI chemotherapy (temozolomideirinotecan) until December 2013. She is currently alive with no evidence of disease, more than 4 years following diagnosis.
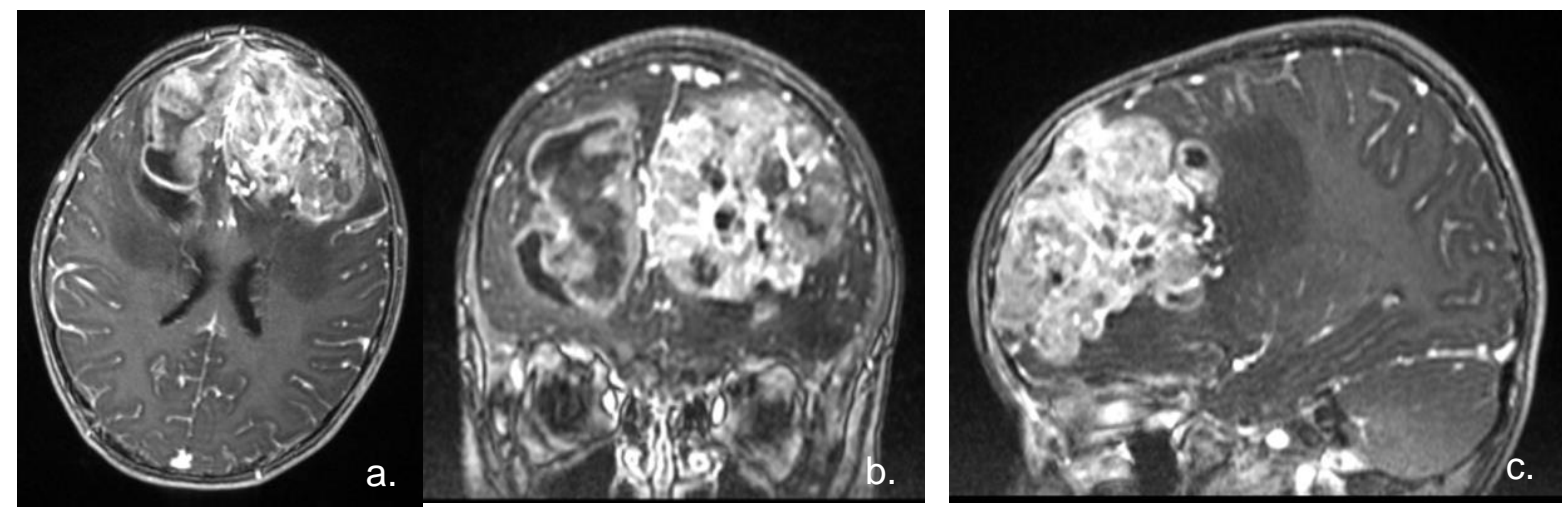

Fig. 1. Sections of brain T1 gadolinium MRI: a. Axial, b. Coronal and c. Sagittal

\section{Discussion}

Ewing's sarcoma are the second most frequent primary bone tumors after osteosarcomas, and represent about 3\% of all cancers in children. Twenty to $30 \%$ of cases are diagnosed in the first decade with a male:female incidence ratio of approximately 1.22 [1]. Contrary to osteosarcomas, ES commonly involves flat bones in a proportion as high as $50 \%$ [1]. In these cases, the main locations are pelvis (26\%), chest wall (16\%), while the skull location is only $2 \%$. In some cases, ES occurs as a primary soft tissue neoplasm without involvement of the bone and is then referred to as extraosseous ES. In extremely rare instances, these lesions are located within the brain parenchyma where they have been often misdiagnosed as cerebral c-PNET. It is noteworthy that nowadays PNET are defined as embryonal tumors according to the 2016 version of the OMS brain tumors classification [2]. As far as our knowledge goes, less than twenty cases of the central nervous system extraosseous Ewing's sarcoma (CNS-EES) have been reported in pathology literature [3-13]. While CNS-EES could be misdiagnosed as cPNETs, epidemiology, behavior, treatment, and prognosis are significantly different. First of all, while both cancers could be disseminated at initial presentation, it is neither in the same proportion nor the same metastatic locations. In the case of c-PNETs the frequency of metastasis is about $35 \%$, always situated within the CNS [14]. More than $70 \%$ of all pediatric medulloblastoma patients are diagnosed in children under age 10. On the contrary, CNS-EES patients presented metastasis in $25 \%$ of the cases with the most common sites being lung (38\%), bone (31\%), and bone marrow (11\%). Among 19 cases of children's CNS-EES reported in the literature, only 9 appeared clearly to be located within the brain parenchyma [3-9], the other cases being classified as extra-axial. The majority of these cases [6] were located in the frontal lobe, like our case, one was fronto-parietal, one temporo-parietal, and the last one in the posterior fossa. The median age was 7 years (ranging from 2 to 17 years) and 2 patients had pulmonary metastasis at 
diagnosis [3, 5]. In most cases of localized ESS, the treatment combines maximum safe resection and chemotherapy commonly using a combination of vincristine-doxorubicincyclophosphamide, alternating with ifosfamideetoposide, followed by radiation therapy of the primary site and eventually surgery in case of residual disease. Then, the treatment is completed with a less aggressive combination of chemotherapy used in Ewing's sarcoma protocols, for a total of about 8 months. Almost all the children suffering from CNS-EES reported in literature have been treated with chemotherapy and radiotherapy like those treated for C-PNETs. The major difference consisted in the fields of radiotherapy since it is focused on the tumor bed for CNS-EES while craniospinal irradiations were used for cPNETs, leading to much more severe endocrine and neurocognitive late effects [1516]. Among the 8 children harboring a parenchymal CNS-EES for whom treatment was reported in literature, 4 received radiotherapy and chemotherapy, 2 received only radiotherapy, and one only chemotherapy. The only case of leptomeningeal relapse occurred in a patient treated with both radiotherapy and chemotherapy. The prognosis of CNS-EES is obviously unknown, due to their rarity. However, it has been suggested that patients with CNS-EES may have a more favorable outcome than patients with c-PNET, which seems to be confirmed in our case. In the case

\section{References}

1. Bernstein $M$, Kovar $H$, Paulussen $M$, et al. Ewing's sarcoma family of tumors: current management. Oncologist 2006; 11:503-519.

2. Louis DN, Ohgaki $H$, Wiestler OD, et al. The 2007 WHO classification of tumors of the central nervous system. Acta Neuropathol 2007; 114:97-109.

3. Mazur MA, Gururangan S, Bridge JA, et al. Intracranial Ewing's sarcoma. Pediatr Blood Cancer 2005; 45:850-856.

4. VandenHeuvel KA, Al-Rohil RN, Stevenson $\mathrm{ME}$, et al. Primary intracranial Ewing's sarcoma with unusual features. Int J Clin Exp Pathol 2015; 8:260-274. of non-metastatic c-PNET, the 5-year EFS is about $60 \%$. The outcome of CNS-EES is poorly reported among the 8 previously mentioned cases. Indeed, it is not known for the two metastatic patients, one patient had a leptomeningeal relapse without precision about the disease free duration, and the 5 other patients were disease free at the time of publication (at 11, 12, 18, 24, 60 months from diagnosis) [4, 6-8].

\section{Conclusion}

Intraparenchymal CNS-EES are very rare tumors, mostly located within the frontal region often misdiagnosed with other varieties of primary brain tumors, particularly embryonal tumors. Their treatment combines surgery, chemotherapy and focal radiotherapy providing good chance of disease free survival.

\section{Conflict of interest}

The authors declare no conflict of interest.

\section{Patient consent}

Written informed consent was obtained from the patient for publication of this case report and accompanying images. A copy of the written consent is available for review by the Editor-in-Chief of this journal.
5. Pekala JS, Gururangan S, Provenzale JM, et al. Central nervous system extraosseous Ewing sarcoma: radiologic manifestations of this newly defined pathologic entity. $A m \mathrm{~J}$ Neuroradiol 2006; 27:580-583.

6. Kazmi SA, Perry A, Pressey JG, et al. Primary Ewing sarcoma of the brain: a case report and literature review. Diagn Mol Pathol 2007; 16:108-111.

7. Jay V, Zielenska M, Lorenzana A, et al. An unusual cerebellar primitive neuroectodermal tumor with $\mathrm{t}(11 ; 22)$ translocation: pathological and molecular analysis. Pediatr Pathol Lab Med 1999; 16:119-128. 
8. Bunyaratavej $\mathrm{K}$, Khaoroptham S, Phonprasert $\mathrm{C}$, et al. Primary intracranial peripheral primitive neuroectodermal tumor/Ewing's sarcoma presenting with acute intracerebral hemorrhage. Clin Neuropathol 2005; 24:184190.

9. Choudhury KB, Sharma S, Kothari R, et al. Primary extraosseous intracranial Ewing's sarcoma: Case report and literature review. Indian J Med Paediatr Oncol 2011; 32:118-121.

10. Antunes NL, Lellouch-Tubiana A, Kalifa C, et al. Intracranial Ewing sarcoma/'peripheral' primitive neuroectodermal tumor of dural origin with molecular genetic confirmation. $J$ Neurooncol 2001; 51:51-56.

11. Papotti M, Abbona G, Pagani A. Primitive neuroectodermal tumor of the meninges: a histology, immunohistochemical, ultrastructural and cytogenetic study. Endocr Pathol 1998; 3:275-280.

12. Dedeurwaerdere F, Giannini $C$, Sciot $R$, et al. Primary peripheral PNET/Ewing's sarcoma of the dura: a clinicopathologic entity distinct from central PNET. Mod Pathol 2002; 15:673-678.

13. Katayma $Y$, Kimura S, Watanabe $T$, et al. Peripheral-type primitive neuroectodermal tumor arising in the tentorium: case report. $J$ Neurosurg 1999; 90:141-144.

14. Taylor RE, Bailey CC, Robinson $\mathrm{KJ}$, et al. Outcome for patients with metastatic (M2-3) medulloblastoma treated with SIOP/UKCCSG PNET-3 chemotherapy. Eur J Cancer 2005; 41:727-734.

15. Edelstein K, Spiegler BJ, Fung S, et al. Early aging in adult survivors of childhood medulloblastoma: long-term neurocognitive, functional, and physical outcomes. Neuro Oncol 2011; 13:536-545.

16. Laughton SJ, Merchant TE, Sklar CA, et al. Endocrine outcomes for children with embryonal brain tumors after risk-adapted craniospinal and conformal primary-site irradiation and highdose chemotherapy with stem-cell rescue on the SJMB-96 trial. $J$ Clin Oncol 2008; 26:1112-1118. 